\title{
Focused Ion Beam Micromachining Enables Novel Optics for X-ray Microscopy
}

\author{
Kahraman Keskinbora ${ }^{1}$, Umut T. Sanli ${ }^{1}$, Corinne Grévent ${ }^{1}$, Michael Hirscher ${ }^{1}$ and Gisela Schütz ${ }^{1}$ \\ ${ }^{1 .}$ Max Planck Institute for Intelligent Systems, Dept. Modern Magnetic Systems, Stuttgart, Germany
}

X-ray microscopy is a strong analytical tool with a plethora of applications in physics, materials science and life sciences [1]. In many high resolution X-ray microscopes, a focusing optic such as a Fresnel zone plate (FZP) is utilized as a lens to form an image. The FZP is a diffractive optic composed of a series of concentric rings (zones) of radially varying grating period, where the width, $\Delta r$, of the outermost zone determines the resolution. Synchrotron radiation facilities are required as bright and coherent X-ray sources as the FZPs are usually quite limited in diffraction efficiency. The diffraction efficiency depends on FZP properties such as geometry, material, structure thickness and on the outermost zone width for small $\Delta \mathrm{r}$. Conventional FZP profiles are binary and can have up to 10-40 \% diffraction efficiency for ideal absorption and phase reversal zone plates, respectively. In practice the efficiencies are lower due to fabrication difficulties and errors. Electron beam lithography (EBL), the usual fabrication method, allows fabrication of binary FZPs with ultra-high resolution via processes of ever increasing complexity. However, due to the scattering of electrons within the resist, it is very difficult to fabricate ultra-high resolution FZPs with high structural thicknesses to achieve high aspect ratios. This, so called, proximity effect limits the aspect ratio, restricting the utilization of high resolution FZPs to softer X-ray energies. One special type of refractive/diffractive X-ray optic is the kinoform lens, with a continuous 3D surface profile. Ideally, it has a theoretical diffraction efficiency of $100 \%$. While in a real lens absorption would hinder $100 \%$ efficiency, diffraction efficiencies well above $40 \%$ are already demonstrated [2] using these lenses. Nevertheless, in order to fabricate these kinoforms, researchers resorted to step approximations using several consecutive overlay EBL steps, which is complicated and lead to much lower efficiencies than theoretically expected due to the vulnerability of the optics to fabrication errors amplified by error accumulations.

To solve the issues that are intrinsic limitations of the EBL-FZPs we introduced novel, precise and mostly direct methods relying on the FIBs. The powerful method potentially allows for higher resolutions than EBL [3] and precise 3D sculpting capability enables the realization of highly efficient X-ray optics with high fidelity [4]. We have used a standard multi-purpose FIB instrument (Nova 600 NanoLab, DualBeam, FEI) to fabricate binary FZPs, kinoform lenses and multilayer FZPs (Figure 1).

Fabrication of binary FZPs using IBL is a simple process (Figure 1a) which delivers high resolution FZPs in a very short time frame, in the order of $10 \mathrm{~min}$, (e.g. $13 \mathrm{~min}$ [5]). Using these binary FZPs made out of gold, we were able achieve $30 \mathrm{~nm}$ effective $\Delta \mathrm{r}$ and half-pitch X-ray image resolutions down to 21 $\mathrm{nm}$. Further progress down to $25 \mathrm{~nm} \Delta \mathrm{r}$ (50 period), is possible (Figure 2a) via optimization of processes and materials.

Various kinoform lenses were fabricated using a gray-scale IBL approach by taking advantage of the 3D fabrication capability of the FIB instrument (Figure 1b) [4]. It was possible to use these lenses for high efficiency soft X-ray focusing and imaging. The diffraction efficiency up to about $14 \%$ was achieved and was limited by the strong absorption, a characteristic of the soft X-rays. Nevertheless, an experimental diffraction efficiency up to $90 \%$ of the theoretical efficiency was achieved, thanks to the high surface quality of the lens (Figure $2 b$ ) and the high precision fabrication process [4]. 
Furthermore, in another approach, micro-machining and micro-manipulating capabilities of FIBs can also be utilized for high precision slicing, transfer and polishing of the multilayer (ML) FZPs, for both the hard and the soft X-rays (Figure 1c). By using an FIB, slices of a multilayer deposit have been successfully cut out and transferred onto a TEM grid where the surfaces were further polished to a fine optical finish and finally a Pt beamstop was deposited via focused ion beam induced deposition (Figure 1c). The proper function of the ML-FZP (Figure 2c), was demonstrated at a synchrotron radiation facility by resolving $\sim 20 \mathrm{~nm}$ (half-pitch) features, without any apparent astigmatism [6].

\section{References:}

[1] D. T. Attwood, "Soft x-rays and extreme ultraviolet radiation: principles and applications", Cambridge Univ Press, New York (2000).

[2] E. D. Fabrizio, F. Romanato, M. Gentili et al., Nature, 401 (1999), 895-898.

[3] W.-D. Li, W. Wu, and R. S. Williams, J. Vac. Sci. Technol., B, 30, (2012), 06F304-4.

[4] K. Keskinbora, C. Grévent, M. Hirscher et al., Advanced Optical Materials, (2014), doi: 10.1002/adom.201400411.

[5] K. Keskinbora, C. Grévent, U. Eigenthaler et al., ACS Nano, 7 (2013), 9788-9797.

[6] K. Keskinbora, A.-L. Robisch, M. Mayer et al., Optics Express, 22 (2014), 18440-18453.

a)

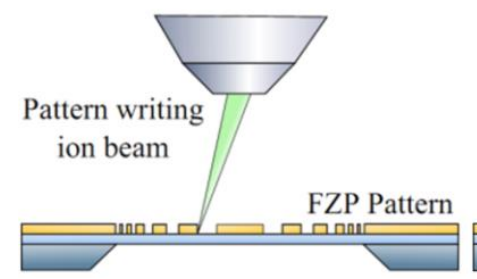

b)

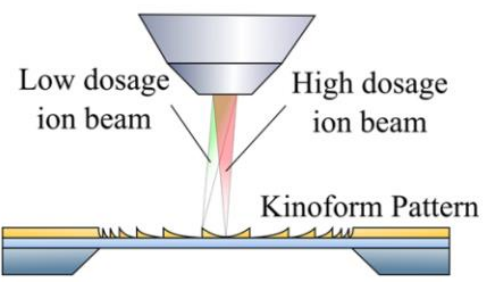

c)

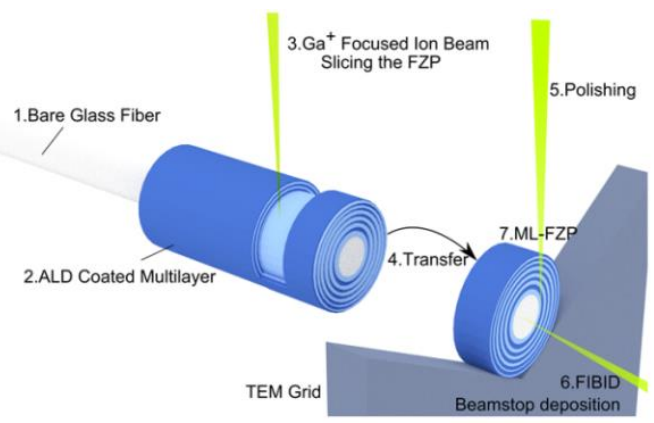

Figure 1. Schematic summary of uses of FIB for X-ray optics manufacturing. a) Single step writing of a binary FZP. b) Single step writing of a kinoform lens using gray-scale IBL. c) Fabrication of the MLFZPs; slicing, transfer, polishing and beamstop deposition are made by using FIB.
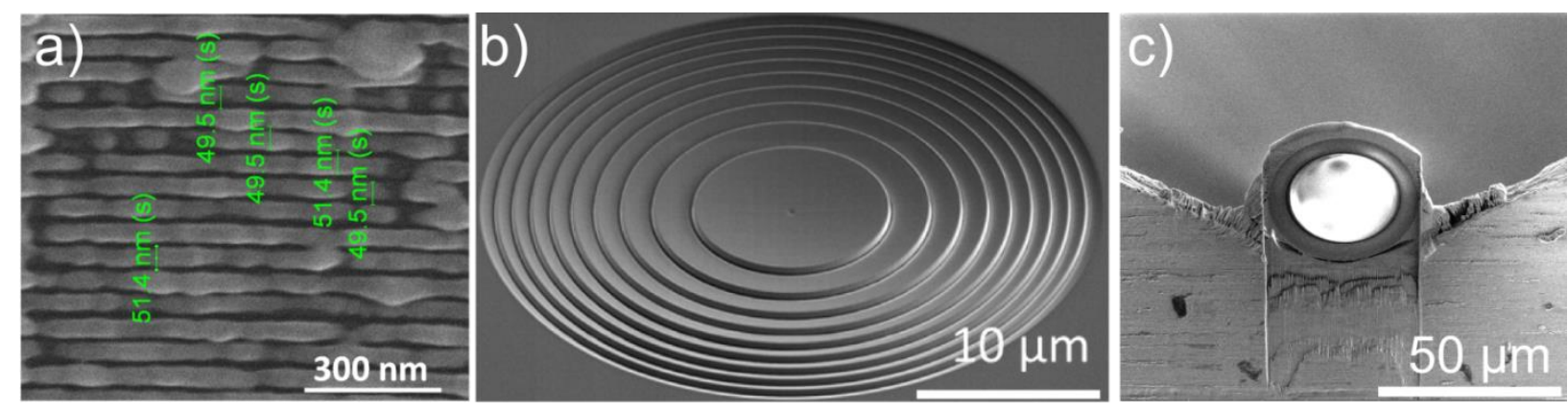

Figure 2. a) Binary zones with $50 \mathrm{~nm}$ period written with a FIB. b) A kinoform lens fabricated by using a gray scale $\mathrm{FIB}$, written in a nano-crystalline $\mathrm{Pd}_{0.8} \mathrm{Si}_{0.2}$ alloy [4]. c) A multilayer FZP transferred onto a TEM grid and polished by FIB, ready for beamstop deposition. 